\title{
The Cultural Aspects of Sharing and Dynamic Partnerships within an SDI Hierarchy
}

\author{
Abbas Rajabifard ${ }^{1}$, Mary-Ellen Feeney ${ }^{2}$ and Ian P. Williamson ${ }^{3}$ \\ ${ }^{1}$ Research Fellow \\ Phone: +61 383440234 Fax: +61393472916 \\ Email: abbas@sunrise.sli.unimelb.edu.au \\ ${ }^{2} \mathrm{PhD}$ Candidate \\ Phone: +61383449696 Fax: +61393472916 \\ Email: $\underline{\text { mef@eng.unimelb.edu.au }}$ \\ ${ }^{3}$ Professor of Surveying and Land Information, \\ Phone: +61 383444431 Fax: +61 393474128 \\ Email: i.williamson@eng.unimelb.edu.au \\ Centre for Spatial Data Infrastructures and Land Administration \\ Department of Geomatics, The University of Melbourne, Victoria 3010, Australia \\ http://www.geom.unimelb.edu.au/research/SDI research/
}

Keywords: Spatial Data Infrastructure (SDI), SDI Hierarchy, Partnerships, Cultural and Social Factors

\begin{abstract}
The need to create multi-participant, decision-supported environments to address the issues of sustainable development and improving the quality of life creates a growing need to organise data across disciplines and organisations through different forms of patial data infrastructure (SDI). This infrastructure is fundamentally a concept about facilitation and coordination of the exchange and sharing of spatial data between stakeholders from different jurisdictional levels in the spatial data community. The concept is well explained as an integrated, multilevelled hierarchy of interconnected SDIs based on partnerships at corporate, local, state/provincial, national, regional (multi-national) and global (GSDI) levels. The creation of such an infrastructure and understanding the role of dynamic partnerships within an SDI hierarchy are essential to develop any SDI initiative.

This paper reviews the nature and concept of SDIs, including the SDI hierarchy, which has helped to build understanding about the importance of the relationships within different levels of SDI, to support the interactions and dynamic nature of partnerships between spatial data communities. The role that human nature plays in any endeavour based on interaction and sharing makes cultural and social factors within a business environment important to the acceptance of the SDI concept and its alignment with spatial industry objectives. Further, the importance of establishing a culture for sharing as well as understanding the dynamic partnerships nec essary to support such a culture is highlighted, and three classes of factors influencing SDI development are identified. It is argued that the adoption and implementation of these factors and selection of a proper model for SDI development can assist SDI coordinating agencies to overcome the problem of low participation and speed up the progress in the development of SDI initiatives.
\end{abstract}




\section{INTRODUCTION}

The growing need to organise data across different disciplines and organisations has resulted in the concept of spatial data infrastructure (SDI). SDI is an initiative intended to create an environment in which all stakeholders can cooperate with each other and interact with technology, to better achieve their objectives at different political/administrative levels. With this in mind, many countries are developing SDIs to better manage and utilise their spatial data assets by taking a perspective that starts at a local level and proceeds through state, national and regional levels to the global (GSDI) level. This has resulted in the development of different forms of SDI at and between these levels. Increasingly, these countries are finding it necessary to cooperate with other countries to develop multi-national SDIs (Regional and Global SDIs) to assist in decision-making that has an important impact across national boundaries. However, current research shows that despite considerable interest and activities, the development of an effective and comprehensive SDI is hampered in most cases by a lack of support from members, which results in this initiative remaining an innovative concept.

This paper aims to address the minimisation of this problem based on the results of a case study and survey research on Regional SDI development in the Asia-Pacific region. It is proposed that this problem can be minimised by increasing the level of awareness about the nature and value of SDIs; understanding the dynamic partnerships which are necessary to support a culture for sharing; improving the SDI conceptual model to better meet the needs of communities; and by identifying key factors that facilitate development by better understanding the complexity of the interaction between social, economic and political issues.

To achieve this aim, the concept and nature of SDIs is discussed in order to facilitate their development and progressive uptake and utilisation by different jurisdictions. This includes the SDI hierarchy, which has helped to build understanding about the importance of the relationships within different levels of SDI, to support the interactions and dynamic nature of partnerships of spatial data communities. The paper then presents three major classes of factors to facilitate the development of an SDI initiative. Finally, two models are reviewed which are thought to provide a new perspective on SDI development, namely a product-based and process-based model. It is argued that the adoption and implementation of these factors and selection of a proper model for SDI development can assist SDI coordinating agencies to overcome the problem of low participation and speed up the progress in the development of SDI initiatives.

\section{SDI - NATURE AND COMPONENTS}

Spatial Data Infrastructure (SDI) is fundamentally a concept about facilitation and coordination of the exchange and sharing of spatial data between stakeholders from different jurisdictional levels in the spatial data community. SDIs have become very important in determining the way in which spatial data are used throughout an organisation, a nation, different regions and the world. In principle, they allow the sharing of data, which is extremely useful, as it enables users to save resources, time and effort when trying to acquire new datasets by avoiding duplication of expenses associated with generation and maintenance of data and their integration with other datasets. By reducing duplication and facilitating 
integration and development of new and innovative business applications, SDIs can produce significant human and resource savings not to mention returns.

An SDI encompasses the policies, technologies, standards and human resources necessary for the effective collection, management, access, delivery and utilisation of geospatial data for a specific jurisdiction or community. Moreover, an SDI is seen as basic infrastructure, like roads, railways and electricity distribution, which supports sustainable development, and in particular economic development, environmental management and social stability (PCGIAP 1998). It is suggested that an SDI comprises not only the four basic components identified by some jurisdictions, like Australia and the Asia-Pacific region (ANZLIC 1996, PCGIAP 1998), but also an important additional component, namely, people (Coleman and McLaughlin 1998, Rajabifard et al. 2002). This component includes the spatial data users and suppliers and any value-adding agents in between, who interact to drive the development of the SDI.

People are the key to transaction processing and decision-making. All decisions require data and as data becomes more valuable and sensitive human issues of data sharing, security, accuracy and access forge the need for more defined relationships between people and data.

Viewing the core components of SDI as policy, access networks, technical standards, people (including partnerships) and data, different categories can be formed based on the different nature of their interactions within the SDI framework. Considering the important and fundamental role between people and data as one category, a second can be considered consisting of the main technological components: the access networks, policy and standards. The nature of both categories is very dynamic due to the changes occurring in communities (people) and their needs, as well as their ongoing requirement for different sets of data. Additionally, with the rapidity with which technology develops, the need for the mediation of rights, restrictions and responsibilities between people and data are also constantly subject to change (Figure 1). This suggests an integrated SDI cannot be composed of spatial data, valueadded services and end-users alone, but instead involves other important issues regarding interoperability, policies and networks. This in turn reflects the dynamic nature of the whole SDI concept. This is an issue which is also highlighted by Groot and McLaughlin (2000).

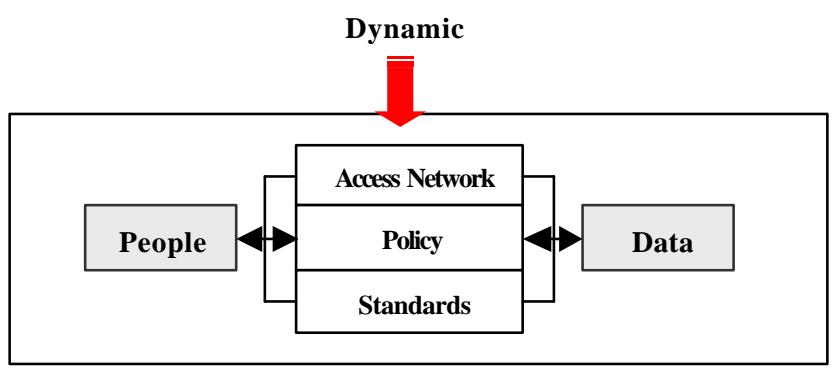

Figure 1: Nature of and relations between SDIs components

According to Figure 1, anyone (data users through producers) wishing to access datasets must utilise the technological components. The influence of the level of SDI and the focus for the technical components have an important influence on the approach taken for aligning components towards the development of SDIs. 
There are numerous approaches taken through varying SDI initiatives for the relationships defined between people, data and the initiative's objectives. One approach has been the development of strategic partnerships. The influence of the level of SDI and the focus for the technical components have an important influence on the approach taken for aligning components towards the development of SDIs.

\section{SDI HIERARCHY}

As a result of developing SDIs at different political/administrative levels, a model of SDI hierarchy that includes SDIs developed at different political-administrative levels was developed and introduced (Rajabifard et al. 2000). Figure 2 illustrates this model in which an SDI hierarchy is made up of inter-connected SDIs at corporate, local, state/provincial, national, regional and global levels. In the model, a corporate SDI is deemed to be an SDI at the corporate level - the base level of the hierarchy. Each SDI at the local level or above is primarily formed by the integration of spatial data sets originally developed for use in corporations operating at that level and below.

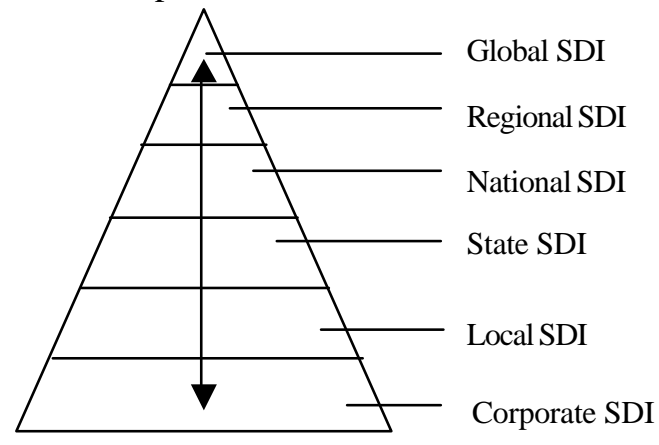

Figure 2. An SDI Hierarchy of SDIs at different levels of Jurisdictions

Understanding the SDI hierarchy is important because it can help to build understanding about the importance of the relationships within different levels of SDI, to support the interactions and dynamic nature of partnerships of spatial data communities. In additional to the vertical relationships between different SDI levels as shown in Figure 2, there are also complex relationships between SDIs within a political/administrative level, at an 'horizontal' level, of an SDI hierarchy which need to be realised as well (Rajabifard et al. 2000). The vertical and the horizontal relationships within an SDI hierarchy are very complex, and this complexity is due to the dynamic, inter- and intra-jurisdictional nature of SDIs (Rajabifard and Williamson 2002). Therefore, in order to map these relationships and take the benefits of SDI hierarchy, any SDI practitioners need to understand the role of dynamic partnerships as they are essential to develop any SDI initiatives.

\section{SDI PARTNERSHIPS AND GLOBAL DRIVERS}

As discussed before, the design of any SDI requires understanding the nature of the concept, the contributing components and the impact of global drivers. Apart from rapid advances in information and communication technologies, the need to define the concept of SDI is justified by drivers such as globalisation, sustainable development, economic reform, political unrest and war, urbanisation, environmental awareness and human rights (Williamson 2000). Moreover, it is the needs of the user community that drive SDI development. These present significant influences on the changing spatial data relationships within the context of SDI jurisdictions. Reliable information infrastructures are needed to record environmental, social and economic rights, restrictions and responsibilities as well as to provide spatial data to facilitate appropriate decision-making and support conflict resolution. These drivers in turn 
effect the resulting spatial data industry environment and SDI vision, in particular the partnership concept.

There has been a trend for countries to expand their efforts in developing SDIs through partnerships, as data sharing is crucial to the success of SDIs. In the 1990s National SDI development took a broad-base approach to encourage cooperation among stakeholders to pool data assets. Based on this approach, an ideal SDI should have all datasets in the corporate SDI fully integrated. Constrained by existing technical and institutional arrangements, SDI developing agencies have focused on promoting adoption of common standards, as well as fast-tracking integration among certain strategic datasets through partnership arrangements (ANZLIC 1996, Jacoby et al. 2001). Partnerships are formed to create business consortia to develop specific data products or services for strategic users, by adopting a focussed approach to SDI development. It is also important to identify and understand the human and community issues as they help to better define and arrange relationships through the partnerships concept.

\section{HUMAN AND COMMUNITY ISSUES}

It is essential that SDI practitioners understand the significance of human and community issues, as much as technical issues, as they determine the success of SDI developments. Community issues determine the long running success of an SDI innovation. SDI, therefore, can no longer be regarded, or taught, primarily as just a technical matter. Developing a successful SDI initiative depends at least as much upon issues such as political support within the community, clarifying the business objectives which the SDI is expected to achieve, sustaining a culture of sharing, maintaining reliable financial support and enlisting the cooperation of all members of the community, as upon technical issues relating to spatial data access, networking, and standards. Therefore, the argument in this paper, is that developing a successful SDI within a jurisdictional level must be seen as a socio-technical, rather than a purely technical, exercise; the communities concerned are expecting to reap benefits from their investment in SDI in terms of improved corporate performances and cooperation.

In the mainstream Information Systems industry for example, it has long been recognised (as noted by Petch and Reeve 1999) that systems fail as much for human as for technical reasons and this reality is increasingly being recognised within the GIS community. Based on Campbell and Masser (1995), 27.2\% of the significant problems that they had experienced were organisationally based, mentioning most frequently poor GIS management structures, staff limitations and lack of support from senior staff.

If the success rate of SDI initiatives is to be improved it is clear that attention needs to be paid to understanding the community and organisational issues within which SDI is supposed to be developed. The SDI initiative has evolved from information systems and techology. With this in mind, as Campbell and Masser (1995) noted that the technological imperative leads us to believe that the adoption of new technology is inevitable and the sooner we adopt the new products the greater will be our advantage. Thus, like any new technology, any community which adopts SDI sooner, will experience greater advantages.

When information systems do fail, as highlighted by Petch and Reeve (1999), the immediate, almost instinctive reaction is to look for technical explanations. What went wrong is that the software couldn't cope, network infrastructures or protocols were inadequate or the response 
times of the system were poor. We regard information systems as technical projects, therefore we look for technical reasons for failure. Furthermore, the recommended remedy will probably involve more of the same; more, newer and better technology will solve the problem.

For many years, however, some computer professionals have concluded that the major reasons for the lack of success of their systems lie not so much in any technical limitations, but rather in their neglect of the human and community aspects of technology (Budic and Godschalk 1996). In short, information systems have been driven by technology-push rather than by demand-pull, (Petch and Reeve 1999). Coleman and McLaughlin (1998) have made the same criticism of the development of SDI initiatives.

In response to the recognition of these problems, Petch and Reeve (1999) believe a sociotechnical viewpoint has increasingly been adopted with the definition of an information system being expanded to include not only the hardware and software, but also the people involved. This viewpoint is as relevant to the development of SDI initiatives and suggests the need to include people, alongside technical and institutional components, in the definition of SDI initiatives throughout the spatial data community. Figure 3, illustrates a socio-technical viewpoint in which a shift is suggested from a techno-centric position to a socio-technical position within a spatial data community.

\begin{tabular}{|ll|}
\hline \multicolumn{2}{|c|}{ Techno-Centric } \\
Spatial data community \\
Focus on technology \\
- $\quad$ Technology push \\
- $\quad$ Because it's possible \\
- $\quad$ Others are developing \\
- $\quad$ Specified by technologist \\
- & Static in nature \\
\hline
\end{tabular}

\begin{tabular}{|c|}
\hline $\begin{array}{c}\text { Socio-Technical } \\
\text { Spatial data community }\end{array}$ \\
\hline 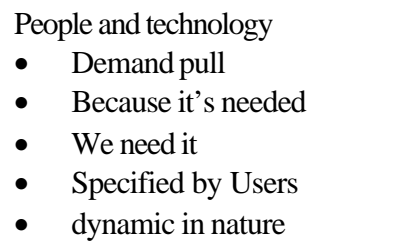 \\
\hline
\end{tabular}

Figure 3: From a techno-centric position to a socio-technical position (Modified from Petch and Reeve 1999)

A socio-technical conception of SDI should emerge in which it is recognised that even if it is assumed that SDI succeeds on a technical level, its adoption still will ultimately depend on how well implementation strategies address the respective community barriers. Recognising that societal issues can be critical factors in determining the success of SDIs, has meant that the SDI's coordinating agency has had to develop a much richer conception of who its community are, how they behave, and particularly how they are likely to respond to the introduction of such a new spatial infrastructure initiative.

With this in mind, Campbell and Masser (1995) provide a convenient tripartite summary of the evolution of attitudes towards the communities into which information systems are introduced:

Technological Determinism: here the community is assumed to provide an unproblematic, indeed almost unconsidered, environment into which new systems can be introduced. The inherent superiority of new technology means that the community will inevitably adopt it.

Managerial Rationalism: here it is recognised that introducing new technology will cause some problems of adjustment within the community. The conception of a community, 
however, is still of a rational, almost machine-like, structure which is amenable to logical adjustment. Problems caused by the introduction of new technology, therefore, can be accommodated by logical restructuring of organisational arrangement and procedures.

Social Interactionism: here, communities are viewed as very complex, social structures which cannot be expected to behave rationally. Communities are viewed as being composed of groups of organisations and individuals each with their own motivations and ambitions. In such a conception, the adoption of new technology, no matter how impressive, is by no means assured. Whether an information system is a success will depend upon a complex interaction of, often informal, political and social forces within the host community.

Due to the complexity of social, cultural, political and administrative systems, and also considering individual members' own motivations and ambitions, the third category of the above attitudes is more applicable for the study and the development of an SDI initiative. In this concept, the adoption of an SDI, no matter how impressive, is by no means assured. Whether an SDI is a success will depend upon a complex interaction of all community issues, including those relating to the sharing of information.

\section{CULTURE FOR SHARING INFORMATION}

Knowledge, or finding better ways of doing things, has always been the main source of longterm economic gain (Langdon and Marshall 1998). However, what is new now is that information technology, which includes the personal computer right through to the Internet as well as the use of robotics in industry, has speeded up the shift towards a knowledge-based society. However, much of the potential for the use of knowledge lies in the ways by which knowledge may be shared. This heavily depends upon the culture of a society. All communities and societies have a culture - a system of shared meaning (Langdon and Marshall 1998). Similarly, any initiative or function, including the sharing of information, also has a specific culture which needs to be promoted to prepare the environment for developing/pursuing the specific activity. Whether that culture is weak or strong is important to both a coordinating agency and individual parties. Therefore, sharing knowledge and information requires a specific culture - a culture for sharing.

Culture is the integrated system of learned patterns of behaviour, ideas, and products characteristic of a society. It is also the ideas people have about their world. In this sense, culture is our mental map of our world and it is also a map for determining action and it becomes a guide for behaviour and decision-making. Langdon and Marshall (1998) view the organisational culture as a shared system of beliefs and values, as evident in rituals, stories, myths and often a specialised language, that fosters a feeling of belonging. They believe an organisation's culture is made up of two elements:

- The organisation's system of shared meaning - shared beliefs and values; and

- The organisation's behavioural norms - how we do things here.

Cultural differences have also persisted between countries at similar stages in industrialisation, and it has been suggested that national culture had a pervasive influence even upon institutional, productive and managerial structures that had formerly been cited as instrumental in international organisational convergence (Neal 1998). 
The debate about the nature of culture has a long history and has been at the heart of sociological, historical and anthropological debates about the relationship between individual action and the nature of society.

Neal (1998) believes, one common lesson propounded by all the researchers is that the relationship between culture and economy is an inter-dependent: a particular form of economy can only persist if it is sustained by a validating culture(s); on the other hand, a particular form of culture can only persist if its ideological and institutional needs are met by a particular form of economy.

Further he reported that, managers find that they are unable to isolate the cultural factors in the diffusion of a new technology and therefore have real problems in trying to manage it (Neal 1998). This is not surprising for in targeting national cultural orders, the complex social factors that are collapsed into the notion of national culture go unaddressed. Cultural problems are the result of complex social dynamics at the interactive and institutional levels. Attempting to manage such problems by targeting transcendent cultural orders overlooks the reasons for these problems.

Many researchers suggest that national cultural differences for example, have an important impact upon the relationship between different countries within a region and their cooperation (Bartlett and Ghoshal 1990, Hennart 1989, Neal 1998).

\section{INFLUENCING FACTORS FOR SDI DEVELOPMENT}

The challenge of designing, building, implementing, and maintaining an SDI draws on many different disciplines and requires examination of a large number of factors and issues. It is essential that SDI practitioners understand the significance of human and societal issues as much as technical issues, all of which contribute to the success of SDI developments. However, sustaining a culture of sharing, a shared language, a shared sense of purpose, and reliable financing is a complex task fraught with difficulties.

For example, Asia and the Pacific region which is one of the first two regions in the world that has started to develop an SDI for its regional level (and the case study of the research) has a complex social and political environment, typified by competing and often conflicting priorities and motivations. Every case in this region is unique because of its national context, language and characteristics (such as size, population, political systems, varied infrastructures and skills), the national traditional and cultural attitudes, and the people who participate, develop and use SDIs.

Therefore, in order to develop a functioning Regional SDI efficiently in this region, the Regional SDI coordinating agency must manage such diversity to gain the support necessary to meet their objectives. Identifying critical social factors and processes in the acquisition, implementation, and utilisation of a technology can facilitate the management of such diversity. It is expected the that decision-making responses of individual nations, groups, and regional organisations may be predicted and therefore may also be accommodated or redirected through prescriptive strategies. 
By identifying key human and technical factors within classes of potential users, SDI coordinating agencies will be able to better define and develop their strategies to achieve their objectives. Having said that, this research has identified three major classes of factors, which are influencing, or contributing to the development of the Asia-Pacific Regional SDI initiative as an example. These classes of factors are Environmental Factors, Capacity Factors, and SDI Organisation Factors, as illustrated in Figure 4. According to the following figure, the three classes of factors together effect the participation rate. The next section will elaborate on each of the classes of factors and their influence on SDI development.

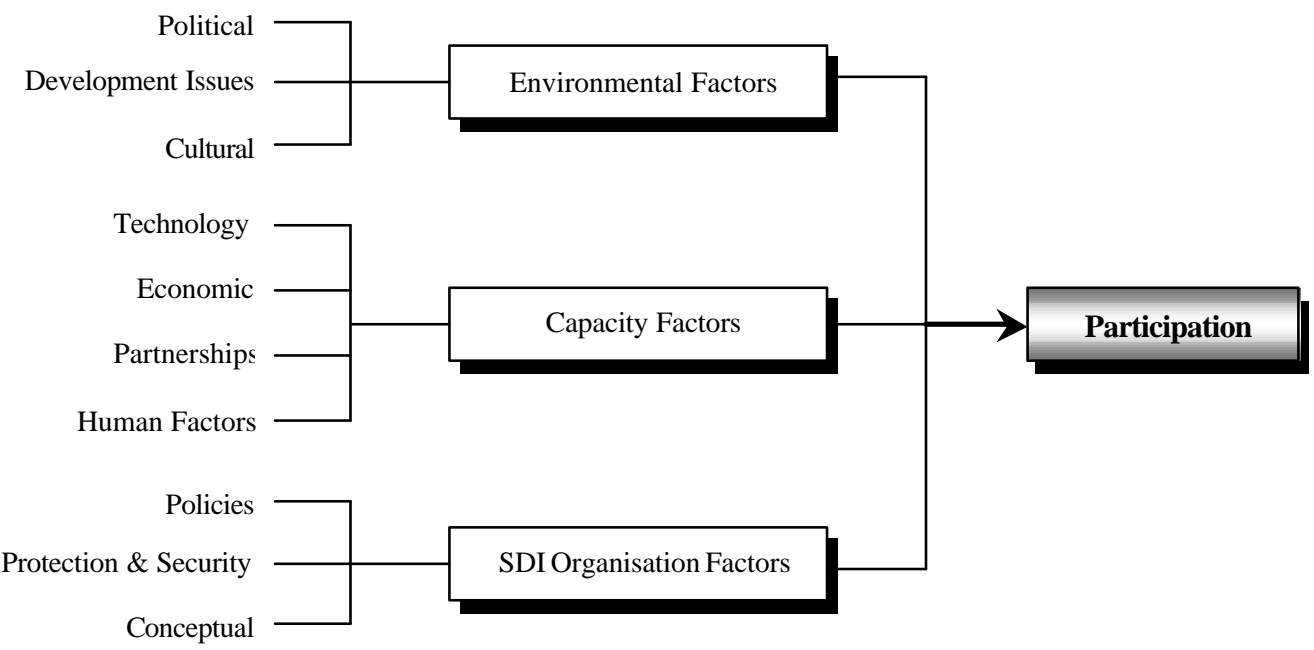

Figure 4: Factors influencing the development of a Regional SDI

\section{A) Environmental Factors}

The environment is the overall structure within which the social system operates and is characterised by internal and external factors. Therefore, the different characteristics of social systems, or communities, adopting the SDI concept can be attributed to a number of environmental factors, including the different cultures of the communities, political factors, and development issues. The external factors are those factors outside the border of the social system which affect, or could potentially affect, the performance of an organisation. These factors impinge more on management levels. The internal factors are those factors inside the border and affect both management and member levels. Therefore, determining an appropriate social border for study and analysis of a social system is very important (understanding the social system is the first step. It determines how we define implementation success and the drivers of implementation success).

Some examples of external factors are Globalisation (global market, global economics, other global initiatives); the GSDI initiative; and the global environment. In terms of internal factors, examples include the political climate; political structure and procedures; relationships with regional organisations; technological pressure; financial stability of each member nation; organisational structure of the coordinating agency (this is one of the most important factors); market pressure; and the degree of culture of data sharing. 
In terms of effectiveness of factors, the effects of cultural factors on Regional SDI development can be extremely high. The social dynamics of national relations can cause enormous effects on cooperation and costs within the business environment and poor decision-making. By examining the social dynamics of cultural difference within jurisdictions, it would be possible to understand why a high proportion of capabilities of member nations is hidden or not functioning. Regional SDI coordinating agencies must therefore assess the impact of cultural factors in SDI development. If the risk of these factors is seen to be too high, then the strategy for development can be redesigned. This can be done through human resource policy, selection of a conceptual model, training and language schemes.

Moreover, the cultural factors should no longer be treated as intangible. They can be analysed and they can be incorporated into the study of the social system of a jurisdiction in which an SDI is supposed to be developed. These are factors of enormous significance in determining the level of support and expectation of individual members within a specific jurisdiction. Those who want to increase the level of support should therefore consider these factors carefully. Thus, any SDI coordinating agency must consider cultural factors to gain significant progress in their activities through concerted policies.

\section{B) Capacity Factors}

Capacity building as defined by Georgiadou (2001), may refer to improvements in the ability of institutions and (government and non-government) organisations to carry out their functions and achieve desired results over time. It may also refer to the provision of foundation data, metadata standards, clearinghouse functionalities and a facilitating environment for decentralising GIS application in manageable application domains within the SDI concept. Therefore, based on this definition, capacity building for an SDI in a broad sense may refer to improvements in the ability of all involved parties to perform appropriate tasks within the broad set of principles of that particular SDI initiative.

Capacity building can be undertaken in various ways, but needs to take into account both institutional as well as individual level capacity building. In this regard, the importance of training in creating a successful environment for SDI diffusion needs to be realised. Training should be of the largest possible breadth and depth. It is not simply a matter of learning a particular concept. It goes much further than that, to a whole new way of thinking about sharing and exchanging spatial data assets, and about optimum solutions. So this is an essential and important issue to be considered for the success of an SDI diffusion.

With this in mind, Capacity Factors are those that cover technology, economic factors, partnerships, and human factors and thus encompass technological capacity, human capacity, and financial capacity. Some examples of Capacity Factors are: the level of awareness of values of SDIs; the state of infrastructure and communications; technology pressures; the economic and financial stability of each member nation (including the ability to cover participation expenses); the necessity for long-term investment plans; regional market pressures (the state of regional markets and proximity to other markets); the availability of resources (lack of funding can be a stimulus for building partnerships, however, there should be a stable source of funding); and the continued building of business processes. 


\section{C) SDI Organisation Factors}

These are factors related to the way that an SDI is defined, designed and implemented. This mainly includes all SDI core components, including technical and institutional issues such as access policies, access networks, technical standards, and the SDI conceptual model. Some examples of SDI Organisation Factors are: the suitability and degree of complexity of the SDI conceptual model; the availability of spatial data and metadata; the integration and inter-flow of datasets from different parties (this has important implications for the ownership and control of information); access networks; and multiple trusted data sources.

As discussed earlier in this paper, much has been done to describe and understand the components and interactions of different aspects of SDIs and their integration into the transactions of the spatial data community. However, there is still a need for descriptions to actually represent the discrepancies between the role and deliverables of an SDI and thus contribute to a simpler, but dynamic, understanding of the complexity of the SDI concept. To this end, Rajabifard et al. (2002) suggested, that the roles of SDI have been pursued through two different approaches: product-based and process-based models, which contribute to the evolution, uptake and utilisation of the SDI concept in different ways.

The productbased model, represents the main aim of an SDI initiative being to link existing and potential databases of the respective political/administrative levels of the community. Whilst, the process-based model, presents the main aim of an SDI initiative as defining a framework to facilitate the management of information assets. In other words, the objectives behind the design of an SDI, by any coordinating agency, are to provide better communication channels for the community for sharing and using data assets, instead of aiming toward the linkage of available databases. In return, this can also facilitate the concept of partnerships.

The process-based model emphasises the communication channel of knowledge infrastructure and capacity building. With this in mind, an SDI initiative can proceed by following certain steps towards the creation of an infrastructure in which to facilitate all parties of the spatial data community in the cooperation and exchange of their datasets. This facilitation can be supported by creating a clearinghouse system, metadata directory system or other forms of collecting and storing information about datasets and databases within that community. This is the prerequisite for data discovery and access. By creating such systems the coordinating agency is able to increase the knowledge infrastructure for that community by which to enable them to better identify appropriate datasets and communication links with custodial agencies. In order to take full advantage of this approach, it is important to understand the social system of the community in which this approach is designed to be executed.

Based on these two SDI development models, it is possible to classify different levels of an SDI hierarchy, according to the roles played within different political/administrative levels and their similarities to the organisational structure, as illustrated in Figure 5. 


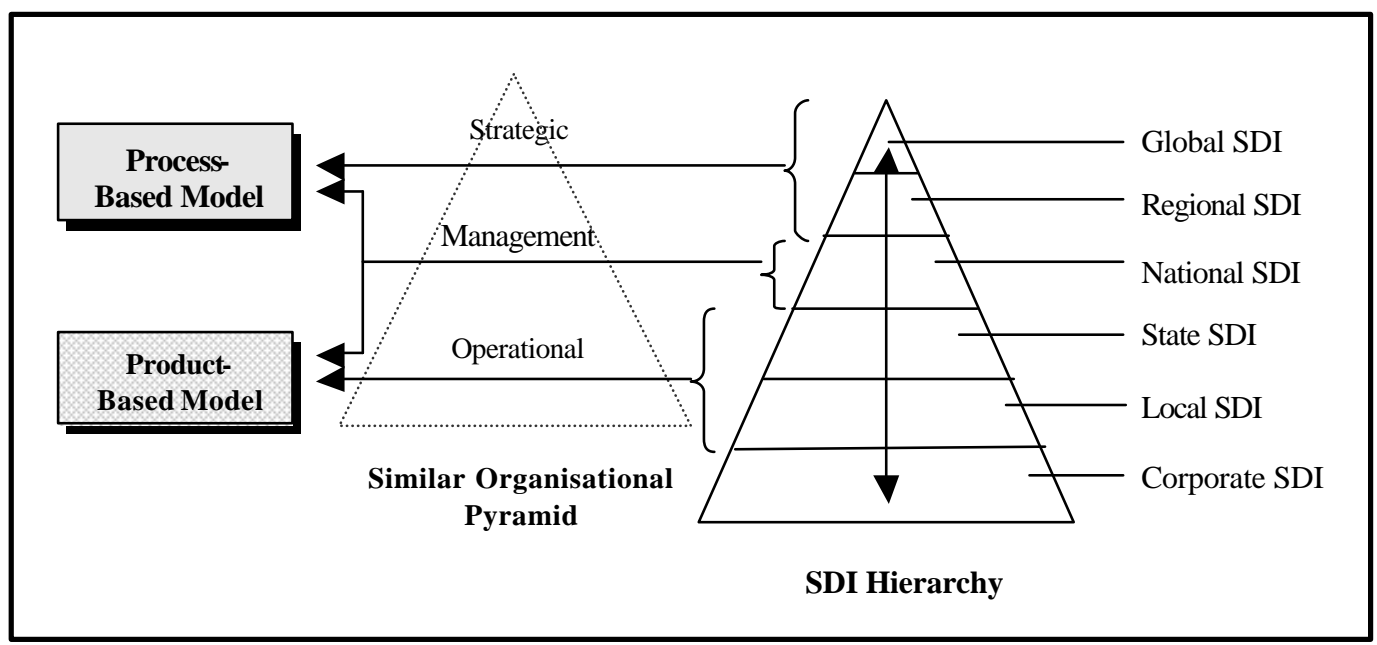

Figure 5: Relationships between SDI Hierarchy and different models of SDI development

According to this figure, and based on the nature of SDIs, any multi-national SDI (regional or global) can be considered similar to the strategic tier of an organisational structure. An SDI at a national level resembles both managerial and strategic tiers, depending on the political system of the nation. If the nation is a federated system of states/provinces (like Australia and USA), then more advantage can be taken from adopting a process-based model to develop a national SDI. Non-federated nations can select a different SDI development model that will optimise their advantage.

The local and state levels of an SDI hierarchy are similar to the operational tier of an organisational structure. Both these levels of SDIs produce data and thus form data which contributes to higher levels of an SDI hierarchy. However, state level SDIs can play a more important role in a federal system of government, because due to the power and responsibilities of states, state-level SDIs can emulate management or operational organisational tiers, or both, for the entire state. Both management and operational tiers take product-based models due to their key roles in data development. Only the strategic tier and nations with federal systems are advised to adopt the process-based model of SDI development. The main reason multinational and federated nations can benefit more from using a process-based model is that SDI participation at these levels of SDI hierarchy is voluntary.

\section{CONCLUSION}

This paper discussed and presented the key factors influencing the development of an SDI, which has been part of the outcome of ongoing research. The paper first reviewed the nature and the concept of SDIs, including the SDI hierarchy, which helped to build understanding about the importance of the relationships within different levels of SDI, to support the interactions and dynamic nature of partnerships of spatial data communities. Then, it discussed human and community issues which are important in long running success of an SDI initiative. It further identified key factors influencing the development of SDIs. The paper presented and discussed three major classes of factors, namely Environmental Factors, Capacity Factors, and SDI Organisation Factors, and then argued that these three classes of 
factors are influencing the development of SDIs, and together they may effect the participation rate of members. Future directions of SDI development were then discussed by reviewing two models, which provided a new perspective, namely a productbased and process-based model.

It is argued that the adoption and implementation of these factors and the selection of a proper model can assist the SDI coordinating agencies in such a way that they improve participation rate and speed up their progress in the development of the SDI initiative.

\section{ACKNOWLEDGMENTS}

The author would like to acknowledge the support of the University of Melbourne, the Australian Land Information Group (AUSLIG), the Permanent Committee on GIS Infrastructure for Asia and the Pacific (PCGIAP), and the member of the Centre for Spatial Data Infrastructures and Land Administration at the Department of Geomatics, the University of Melbourne, in the preparation of this paper and the associated research. However, the views expressed in the paper are those of the authors and do not necessarily reflect the views of these groups.

\section{REFERENCES}

ANZLIC (1996). National spatial data infrastructure for Australia and New Zealand, ANZLIC Discussion Paper, Commonwealth of Australia, http://www.anzlic.org.au/anzdiscu.htm, (Accessed November 1998).

Bartlett, C.A. and Ghoshal, S.A. (1990). Managing Across Borders, Hutchinson Business Books, London.

Budic, Z. D. and Godschalk, D. R. (1996). Human Factors in Adoption of Geographic Information Systems: A local Government Case Study, Public Administrative Review, November/December 56(6): 554-567.

Campbell, H. and Masser, I. (1995). GIS and Organizations, London, UK; Bristol, PA, Taylor \& Francis.

Coleman, D. J. and McLaughlin, J.D. (1998). Defining Global Geospatial Data Infrastructure (GGDI): components, stakeholders and interfaces, Geomatica 52(2): 129-144.

Georgiadou, Y. (2001). Capacity Building Aspects for a Geospatial Data Infrastructure (GDI). Proceedings of $5^{\text {th }}$ Global Spatial Data Infrastructure Conference, 21-25 May, Cartagena de Indias Colombia.

Groot, R. and McLaughlin, J. (2000). Geospatial Data Infrastructure: concepts, cases and good practice, Oxford University Press, New York, 286 p.

Hennart, J.F. (1989). Can the 'New' Forms of Investment Sustitute for the Old Forms? A transaction Costs Perspective', Journal of International Business Studies (summer) 211-234.

Jacoby, S., Smith, J., Ting, L., \& Williamson, I. (2001). Forthcoming. Developing a Common Spatial Data Infrastructure between State \& Local Government - An Australian case study, International Journal of GIS.

Langdon, A. and Marshall, P. (1998). Organistional Behaviour, Published by Addison Wesley Longman, Australia.

Neal, M. (1998), The Culture Factor: Cross-National Management and the Foreign Venture, Published by Macmillan Press, London.

PCGIAP (1998). A Spatial Data Infrastructure for the Asia and the Pacific Region, (PCGIAP Publication No. 1, Canberra).

Petch, J. and Reeve, D. (1999). GIS Organisations and People, a socio-technical approach, Taylor \& Francis, UK.

Rajabifard, A. and Williamson, I. P. (2002). Spatial Data Infrastructures: an initiative to facilitate spatial data sharing', F orthcoming Book, Global Environmental Databases, Volume 2, 
International Society for Photogrammetry and Remote Sensing (ISPRSWG IV/8), Chiba, Japan.

Rajabifard, A., Feeney, M., and Williamson I.P. (2002). Future Directions for the Development of Spatial Daa Infrastructure, Forthcoming publication, Journal of the International Institute for Aerospace Survey and Earth Sciences, ITC, The Netherlands.

Rajabifard, A. and Williamson, I.P. (2001). Spatial Data Infrastructures: Concept, SDI Hierarchy and Future directions, Proceedings of GEOMATICS' 80 Conference, 29 April-2 May 2001, Tehran, Iran.

Rajabifard, A. Williamson, I.P., Holland, P., and Johnstone, G. (2000). From Local to Global SDI initiatives: a pyramid building blocks, Proceedings of the $4{ }^{\text {th }}$ Global Spatial Data Infrastructures Conferences, 13-15 March 2000, Cape Town, South Africa.

Williamson, I.P. (2000). Institutional Framework Reforms for Land administration. Topic Cycle 10, World Bank Land Administration Project - Part C. National Development Planning Agency (BAPPENAS) and National Land Agency, Government of the Republic of Indonesia. www.landpolicy.org Accessed August, 2000. 
Figure 1. Nature of and relations between SDIs components

Figure 2. An SDI Hierarchy of SDIs at different levels of Jurisdictions

Figure 3. From a techno-centric position to a socio-technical position (Modified from Petch and Reeve 1999)

Figure 4. Factors influencing the development of a Regional SDI

Figure 5. Relationships between SDI Hierarchy and different models of SDI development 


\section{University Library}

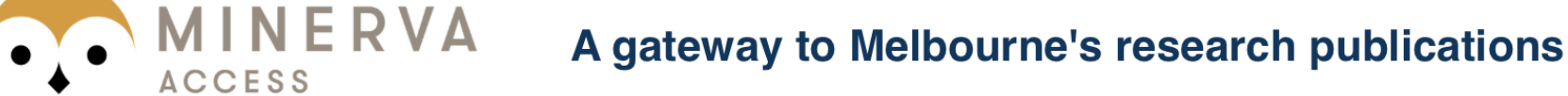

Minerva Access is the Institutional Repository of The University of Melbourne

Author/s:

Rajabifard, A.;Feeney, M-E. F.;Williamson, I. P.

Title:

The Cultural Aspects of Sharing and Dynamic Partnershipswithin an SDI Hierarchy

Date:

2002

Citation:

Rajabifard, A. and Feeney, M-E. F. and Williamson, I. P. (2002) The Cultural Aspects of Sharing and Dynamic Partnershipswithin an SDI Hierarchy, in Proceedings, of MSIA 50th Anniversary Conference, Melbourne, Australia.

Publication Status:

Published

Persistent Link:

http://hdl.handle.net/11343/33877 